\title{
Falsification of Cyber-Physical Systems Through Multi-Fidelity Stochastic Optimization
}

\author{
Alireza Inanlouganji, Shakiba Yaghoubi, Georgios Fainekos, Giulia Pedrielli \\ ainanlou@asu.edu,syaghoub@asu.edu,fainekos@asu.com,gpedriel@asu.edu \\ Arizona State University \\ Tempe, Arizona
}

\begin{abstract}
This talk will review some recent results on requirements driven search-based testing for Cyber-Physical Systems (CPS). Namely, we will present an extension to a Bayesian optimizer that makes use of models of different complexity (and accuracy) that allow obtaining several approximations of the guiding cost function. The goal of the method is to enable falsification of computationally expensive simulation models through the use of simpler but much faster simulation models.
\end{abstract}

\section{CCS CONCEPTS}

- Computer systems organization $\rightarrow$ Embedded systems; Redundancy; Robotics.

\section{KEYWORDS}

optimization, cyber-physical systems, testing, falsification

\section{ACM Reference Format:}

Alireza Inanlouganji, Shakiba Yaghoubi, Georgios Fainekos, Giulia Pedrielli. 2019. Falsification of Cyber-Physical Systems Through MultiFidelity Stochastic Optimization. In SNR '19: Symbolic-Numeric methods for Reasoning about CPS and IoT, April 15, 2019, Montreal, QC. ACM, New York, NY, USA, 2 pages. https://doi.org/10.1145/1122445.1122456

\section{INTRODUCTION \& MOTIVATION}

This work is in the field of requirements driven search-based test case generation methods for Cyber-Physical Systems (CPS) [1]. The basic characteristic of search-based testing methods is that the search process is guided by high level requirements captured in formal logic and, in particular, Signal Temporal Logic (STL) [2]. Given a system trajectory, STL specifications can be equipped with quantitative semantics which evaluate to the distance of the given trajectory from violating the requirement. Hence, by searching for trajectories of decreasing value with respect to the specification, a test generation method can be formulated which searches for system behaviors of value 0 . Such system behaviors, i.e., trajectories that violate the requirements, are referred to as falsifying behaviors. In

Permission to make digital or hard copies of all or part of this work for personal or classroom use is granted without fee provided that copies are not made or distributed for profit or commercial advantage and that copies bear this notice and the full citation on the first page. Copyrights for components of this work owned by others than the author(s) must be honored. Abstracting with credit is permitted. To copy otherwise, or republish, to post on servers or to redistribute to lists, requires prior specific permission and/or a fee. Request permissions from permissions@acm.org.

SNR '19, April 15, 2019, Montreal, QC

(C) 2019 Association for Computing Machinery.

ACM ISBN 978-1-4503-6697-7/19/04 ..\$15.00

https://doi.org/10.1145/3313149.3313371 addition, signed distance can be utilized when searching for trajectories that maximally violate the specification (negative specification valuations). In this work, we present a novel approach that mixes global and local search for improving finite time performance, i.e., quickly identifying falsification inputs.

\section{PROPOSED METHOD}

In our preliminary work [4], we have developed a first extension of a Bayesian optimizer that makes use of models of different complexity (and accuracy) that allow to obtain several approximations of the STL robustness function $R_{\varphi}(\boldsymbol{x})$ $[2,3]$. A multi-fidelity sampler attempts to find the minimizer of the robustness function:

$$
\boldsymbol{x}^{*} \in \underset{\boldsymbol{x} \in \mathbb{X}}{\arg \min } R_{\varphi}(\boldsymbol{x})
$$

where $\mathbb{X}$ represents the input/parameter search for the CPS. In order to solve the general problem in equation (1), we have access to an approximate model of the original system that leads to an inaccurate robustness function. We denote this inaccurate function as $R_{\varphi}^{\ell}(\boldsymbol{x})$ which has a lower associated computational cost when compared to $R_{\varphi}(x)$. The aim is to use the information obtained from sampling within the lowfidelity space to guide the search towards the global optimum of the high-fidelity function $R_{\varphi}(\boldsymbol{x})$. We propose a statistical approach to construct a learning structure link between $R_{\varphi}^{\ell}(\boldsymbol{x})$ and $R_{\varphi}(\boldsymbol{x})$. Formally:

$$
R_{\varphi}\left(\boldsymbol{x} ; \boldsymbol{\theta}_{\boldsymbol{H} F}\right)=R_{\varphi}^{\ell}\left(M\left(\boldsymbol{x} ; \boldsymbol{\theta}_{\boldsymbol{M}}\right) ; \boldsymbol{\theta}_{\boldsymbol{L} F}\right)+B\left(\boldsymbol{x} ; \boldsymbol{\theta}_{\boldsymbol{B}}\right), \boldsymbol{x} \in X .
$$

Under our proposal, $R_{\varphi}^{\ell}$ and $R_{\varphi}$ are both interpreted as realizations of Gaussian processes with $\boldsymbol{\theta}_{L F}$ and $\boldsymbol{\theta}_{\boldsymbol{B}}$ being the corresponding hyper-parameters $[4,6] . M\left(\boldsymbol{x} ; \boldsymbol{\theta}_{\boldsymbol{M}}\right)$ represents a general mapping function from the low-fidelity solution space to the high-fidelity solution space that can help in increasing the similarity between low and high-fidelity models with $\boldsymbol{\theta}_{\boldsymbol{M}}$ being the set of mapping parameters.

The relationship between the approximate and exact robustness function allows to exchange information between the search in low and high fidelity. In particular, we proceed iterating using the low accuracy model which sequentially proposes low accuracy candidates, $x_{k}^{\ell, *} \in \arg \min _{\boldsymbol{x} \in \mathbb{X}^{\ell}} R_{\varphi}^{\ell}(\boldsymbol{x})$. It is important to highlight that using low fidelity information is convenient in sampling since the low fidelity model may be "easier to optimize" and to sample. As an example, we may use random projections and convexification to generate low accuracy representations for the robustness function and quickly produce low accuracy proposals of the type in Eq. (2). But how long can we run the algorithm using $R_{\varphi}^{\ell}(\boldsymbol{x})$ ? We devised a 
statistical certificate of the relationship between the low and high-fidelity models.

For the candidate point at iteration $k, x_{k}^{*}$, we want to certify the validity of our model in Eq. (2) representing the relationship we constructed between low and high-fidelity models. A statistical test can be constructed of the form:

$$
\begin{array}{r}
\mathrm{H}_{0}: \hat{R}_{\varphi}^{\ell}\left(x_{k}^{\ell, *}\right) \sim \mathcal{N}\left(\mu_{\ell}\left(x_{k}^{\ell, *}\right), \sigma_{\ell}\left(x_{k}^{\ell, *}\right)\right) \\
Q \equiv \frac{R^{\ell}\left(x_{k}^{\ell, *}\right)-\mu_{\ell}\left(x_{k}^{\ell, *}\right)}{\sigma_{\ell}\left(x_{k}^{\ell, *}\right)}
\end{array}
$$

where $\mu_{\ell}\left(x_{k}^{\ell, *}\right)$ is obtained using the high fidelity information. Intuitively, the test statistics in equation (4) uses the conditional density of the low fidelity function predicted by the high accuracy robustness to detect whether there has been a change in the assumed model parameters. If the test fails, it means we need to perform expensive high accuracy iterations. In our first empirical evaluation, we observed that calls to the high-fidelity oracle are most likely to happen at the beginning of the procedure and they reduce as the algorithm progresses.

\section{PRELIMINARY RESULTS}

To demonstrate the performance of the approach, we considered the cost functions from [7], with $\boldsymbol{x}_{i} \in(0,1)$ :

$$
\begin{array}{r}
R_{\varphi}(\boldsymbol{x})=-2.5 \prod_{i=1}^{d} \sin \left(\pi x_{i}\right)-\prod_{i=1}^{d} \sin \left(5 \pi x_{i}\right), \\
R_{\varphi}^{\ell}(\boldsymbol{x})=-2 \prod_{i=1}^{d} \sin \left(\pi\left(x_{i}-b\right) / a_{i}\right)
\end{array}
$$

To evaluate the performance of the proposed method, we considered problems with $d=3,6,9,12$ decision variables and $i=1,2, \ldots, 5$ different randomly generated values for $a_{i}$ and $b_{i}$. For each combination of problem size and mapping parameters, 50 macro replications each with a different seed was conducted. The same set of seed values was used to run Efficient Global Optimization (EGO) [5] and the proposed algorithm. The objective is to compare the performance of the algorithm with that of EGO [5] - a state of the art Bayesian optimizer - and to compare the optimality gap achieved upon termination of the algorithm, i.e., $\frac{\left|\hat{R}_{\varphi}^{*}-R_{\varphi}^{*}\right|}{\left|R_{\varphi}^{*}\right|}$ where $R_{\varphi}^{*}$ is the global optimum of the high-fidelity objective and $\hat{R}_{\varphi}^{*}$ is the solution reported by the algorithm upon completion.

Figures 1a-1b show the average optimality gap estimated across 50 macro-replications of the algorithm as a function of the number of high-fidelity estimations performed by the procedure, for the cases in 9 and 12 dimensions. The approach is very promising and, especially for low number of iterations, our approach is orders of magnitude better than the benchmark EGO.

\section{CONCLUSIONS AND DEVELOPMENTS}

Using low fidelity information to guide the search towards the optimum of an expensive high-fidelity model in a multifidelity optimization framework is an issue that recently has

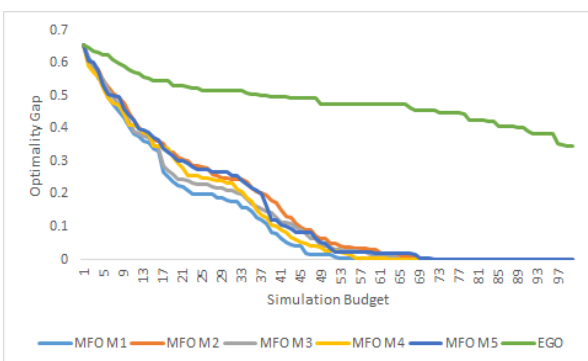

(a) $d=9$

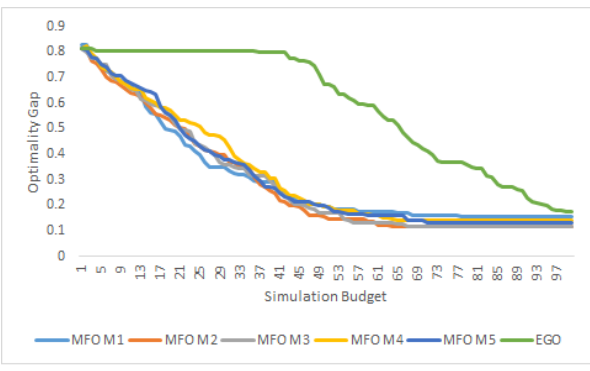

(b) $d=12$

Figure 1: Analysis of Average optimality gap for different dimensions (1a-1b)

attracted much interest in the verification community. In this paper, we introduce a sampler that uses a cheap low fidelity model and uses space mapping to reconcile the information from the low and high fidelity model. We are working on the extension of the framework to different mappings and to enable the automatic generation of low fidelity models.

\section{ACKNOWLEDGMENTS}

This research was partially funded by the awards NSF 1350420 , NSF 1829238, NSF 1361926 and the NSF I/UCRC Center for Embedded Systems.

\section{REFERENCES}

[1] Houssam Abbas, Georgios E. Fainekos, Sriram Sankaranarayanan, Franjo Ivancic, and Aarti Gupta. 2013. Probabilistic Temporal Logic Falsification of Cyber-Physical Systems. ACM Transactions on Embedded Computing Systems 12, s2 (May 2013).

[2] Ezio Bartocci, Jyotirmoy Deshmukh, Alexandre Donzé, Georgios Fainekos, Oded Maler, Dejan Nickovic, and Sriram Sankaranarayanan. 2018. Specification-based Monitoring of Cyber-Physical Systems: A Survey on Theory, Tools and Applications. In Lectures on Runtime Verification Introductory and Advanced Topics. LNCS, Vol. 10457. Springer, 128-168. https://doi.org/10.1007/978-3-319-75632-5_5

[3] Georgios Fainekos and George J. Pappas. 2009. Robustness of Temporal Logic Specifications for Continuous-Time Signals. Theoretical Computer Science 410 (2009), 4262-4291.

[4] Alireza Inanlouganji, Giulia Pedrielli, Georgios Fainekos, and Sebastian Pokutta. 2018. Continuous simulation optimization with model mismatch using Gaussian process regression. In Winter Simulation Conference (WSC). IEEE.

[5] Donald R. Jones, Matthias Schonlau, and William J. Welch. 1998. Efficient Global Optimization of Expensive Black-Box Functions. Fournal of Global optimization 13, 4 (1998), 455-492.

[6] Thomas J Santner, Brian J Williams, and William I Notz. 2013. The Design and Analysis of Computer Experiments. Springer Science \& Business Media.

[7] Zelda B Zabinsky, David Bulger, and Charoenchai Khompatraporn. 2010. Stopping and Restarting Strategy for Stochastic Sequential Search in Global Optimization. fournal of Global Optimization 46, 2 (2010), 273-286. 